\title{
EVALUATION OF FORCE AND PARTICLE VELOCITY AT THE HEATED END OF A ROD SUBJECTED TO IMPACT LOADING
}

\author{
C. BACON, J. CARLSSON ${ }^{(1)}$ and J.L. LATAILLADE \\ Laboratoire de Mécanique Physique, Université de Bordeaux $I$, \\ URA CNRS 867, 351 Cours de la Libération, F-33405 Talence \\ Cedex, France
}

\begin{abstract}
Résumé - Une méthode pour déterminer la force et la vitesse à l'extrémité chauffée d'une barre soumise à des sollicitations dynamiques est présentée. Cette méthode est basée sur le fait que l'impédance peut être représentée par une fonction de plusieurs paramètres. Si la fonction caractéristique d'impédance est connue, la force et la vitesse particulaire à l'extrémité chauffée de la barre peuvent être déterminées à partir des déformations mesurées en deux sections droites de cette barre. Pour évaluer cette fonction, la barre est impactée par un projectile à une extrémité et l'extrémité chauffée est libre. Les paramètres optimaux sont déterminés par une procédure de minimisation de l'erreur, c.a.d. la force à l'extrémité libre de la barre. Les résultats montrent que l'erreur obtenue avec une impédance constante n'est pas négligeable tandis que celle obtenue après minimisation est du même ordre de grandeur que l'erreur pour un essai à température constante. Il est donc possible, avec cette méthode, d'obtenir la force et la vitesse à l'extrémité chauffée d'une barre avec la même précision que pour une barre à température constante.
\end{abstract}

\begin{abstract}
A method to evaluate the force and particle velocity at the heated end of a rod subjected to impact loading is presented. The method is based on a characteristic impedance function determined by a set of parameters. With a given characteristic impedance function the force and particle velocity at the heated end can be evaluated from measured strains at two cross-sections of the rod. In order to evaluate the characteristic impedance function the rod is impacted by a hammer at one end and the heated end is free. The optimal set of parameters is determined by a procedure which minimizes the error, $i$. e. the evaluated force at the free end of the rod. The results show that the error obtained with the constant characteristic impedance function is considerable, whereas the error obtained with the evaluated characteristic impedance function is of the same magnitude as the error obtained for a test at constant temperature. Thus, with this method it is possible to evaluate the force and particle velocity at the heated end of a rod with the same accuracy as for a uniform rod at room temperature.
\end{abstract}

\section{Introduction}

One-dimensional wave propagation in rods forms the basis for several experimental techniques such as, e. g. the split Hopkinson bar. These techniques normally use rods with constant characteristic impedance. Pulses whose Fourier components have wavelengths long compared with the transverse dimension of the rod will propagate without dispersion in such a rod. The force and particle velocity at the end of the rod, i. e. at the specimen, can then easily be evaluated from the incident and reflected pulses in the rod. Problems arise when measurements are to be made at high temperatures. The heating of the specimen results in a non-uniform temperature in the rod. Since the mechanical properties are functions of temperature the characteristic impedance function is also non-uniform. Pulses propagating in a rod heated at one end will be partially, reflected and the pulse shape will be dispersed.

If the temperature distribution in the rod and the relations between the mechanical properties and the temperature are known the problems can be solved by different methods. One method consists of keeping the characteristic impedance constant by a variation of the cross-section area along the rod. This technique has been used by Campbell [1] for a torsional Hopkinson bar. Another method developed by Lundberg, Carlsson and Sundin [2] is based on a known characteristic impedance function and it takes into account the reflections along the rod. With this method the force and particle velocity at the end of a non-uniform rod can be evaluated from measured strains at two cross-sections of the rod. Unfortunately the temperature distribution in the rod is not easily

(1) Visiting scientist. Now at Lulea University of Technology, S-951 87 Lulea, Sweden. 
established and the relation between the characteristic impedance function and the temperature is not well known for common rod materials. The aim of the present study is to determine the characteristic impedance function experimentally without having to establish the temperature distribution.

A direct method to determine the vocal-tract shape from impulse response at the lips has been presented by Sondhi and Gopinath [3] and a generalized solution to determine distributed parameters in lossless wave supporting systems with variable wave propagation speed was presented by Lundberg and Lesser [4]. These methods use the impulse response at one end of the system and the boundary condition at the other end is unknown. In this case it is possible to know the force at the heated end by performing the experiments with a free end.

The present method is an indirect method. The characteristic impedance function is assumed to be a function of a set of parameters. For a given set of parameters the force at the free end of the rod can be evaluated by the method of Lundberg et al. [2]. The optimal set of parameters is determined by a procedure which minimizes the error, $i$. e. the evaluated force at the free end of the rod. The characteristic impedance function evaluated by the method has been used to determine the force and particle velocity at the heated end during impact with a uniform rod. The error can be reduced to the same low level as for tests with rods of constant characteristic impedance. Hence, the method gives accurate values for the evaluated force and particle velocity at the end of a non-uniform rod, with an a priori unknown characteristic impedance function.

\section{Method}

\subsection{Characteristic impedance function}

A linear-elastic rod with a non-uniform temperature distribution $T(x)$, Young's modulus $E(T(x)$ ), density $\rho(T(x))$ and cross-section $A(T(x))$ has the characteristic impedance function

$$
Z(x)=A(T(x))[E(T(x)) \rho(T(x))]^{1 / 2}
$$

This characteristic impedance, $Z$, is determined by a set of parameters and the aim is to find the parameters which minimize the error, $i$. e. the evaluated force at the free end of the main rod. The characteristic impedance function can be evaluated if the following four functions $T=T(x), A=A(T)$, $E=E(T)$ and $\rho=\rho(T)$ are known. The assumed characteristic impedance function is based on the assumptions made for these four functions.

In this study the non-uniform temperature distribution is due to the heating of the end part of the main rod. The temperature, $T(x)$, is assumed to be linear in the part of the main rod inside the furnace, i. e. between the cross-sections $x_{E}$ and $x_{F}$, and to follow the solution of the one-dimensional heat equation between the cross-sections $x_{B}$ and $x_{E}$. The rest of the rod is assumed to be at room temperature, $T_{0}$. The heat flux is assumed to be constant along the rod and the actual temperature distribution has been measured to check the validity of this assumption. These measurements have been made with a thermo-couple (Thermocoax TKI 10/10/DIN) at the bottom of $10 \mathrm{~mm}$ deep holes with a diameter of $1 \mathrm{~mm}$ at the cross-sections indicated in Figure 1, which shows that the actual temperature distribution can be represented by the assumed temperature distribution. Comparison has also been made with the two-dimensional solution but it gives almost exactly the same temperature distribution as the one-dimensional equation in this case. Thus, the assumed temperature $T(x)$ is

$$
T(x)= \begin{cases}T_{0}, & x \leq x_{B}, \\ \left(T_{E}-T_{0}\right) \frac{\sinh \left[\mu\left(x-x_{B}\right)\right]}{\sinh \left[\mu\left(x_{E}-x_{B}\right)\right]}+T_{0}, & x_{B} \leq x \leq x_{E}, \\ \frac{T_{F}\left(x-x_{E}\right)+T_{E}\left(x_{F}-x\right)}{x_{F}-x_{E}}, & x \geq x_{E},\end{cases}
$$

where $T_{E}$ is the temperature at $x_{E}, i$. e. at the entrance of the furnace, and $T_{F}$ is the temperature at $x_{F}$, 
i. e. at the end of the main rod. The parameter $\mu$ is defined as $\mu=2 h / a \lambda$ where $h$ is the heat flux, $\lambda$ the thermal conductivity and $a$ the radius of the rod.

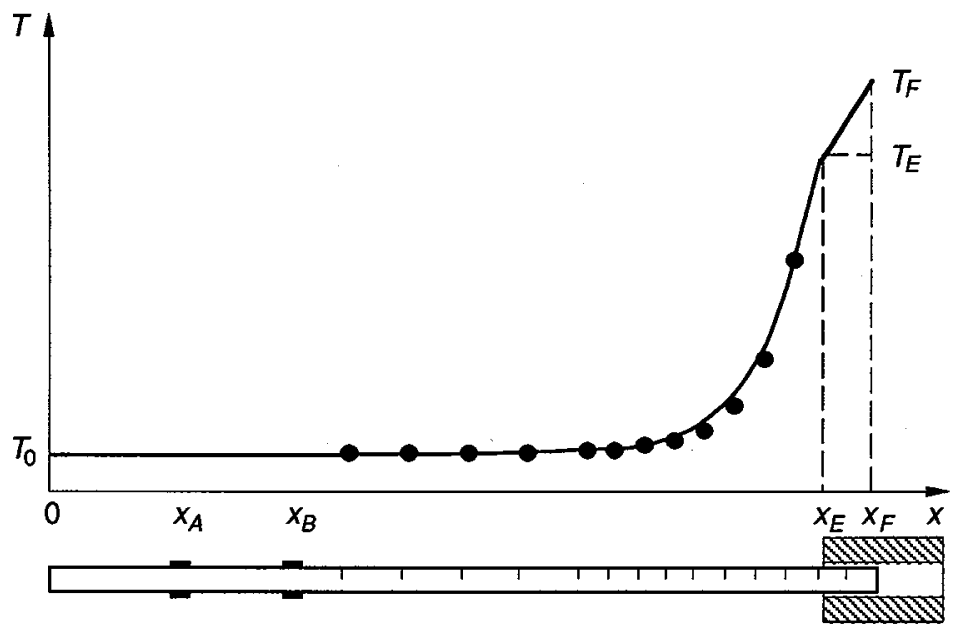

Figure 1. Temperature $T$ vs. axial co-ordinate $x$ with rod end temperature at $950^{\circ} \mathrm{C}$. Measured temperature (dots) and temperature according to 1-D theory (solid line).

In the temperature range between $0{ }^{\circ} \mathrm{C}$ and $1000{ }^{\circ} \mathrm{C}$ the linear thermal expansion coefficient is approximately $17 \cdot 10^{-6} /{ }^{\circ} \mathrm{C}$ and the maximal change in $A(T)[\rho(T)]^{1 / 2}$ is about $9 \%$. Hence, the influence of the thermal expansion on the characteristic impedance has been neglected. to be

The Young's modulus is generally a decreasing function of the temperature and here it is assumed

$$
E(T)=E_{0}\left[1-\beta\left(T-T_{0}\right)\right]
$$

where $E_{0}$ is the Young's modulus at room temperature.

With the assumptions made the characteristic impedance function becomes

$$
Z(x)=Z_{0}\left[1-\beta\left(T(x)-T_{0}\right)\right]^{1 / 2}
$$

where $Z_{0}$ is the characteristic impedance at room temperature and $T(x)$ is given by equation (2). Inserting equation (2) into equation (4) gives

$$
Z(x)= \begin{cases}Z_{0}, & x \leq x_{B}, \\ Z_{0}\left\{1-\beta\left(T_{E}-T_{0}\right) \frac{\sinh \left[\mu\left(x-x_{B}\right)\right]}{\sinh \left[\mu\left(x_{E}-x_{B}\right)\right]}\right\}^{1 / 2}, & x_{B} \leq x \leq x_{E}, \\ Z_{0}\left\{1-\beta \frac{\left(T_{F}-T_{0}\right)\left(x-x_{E}\right)+\left(T_{E}-T_{0}\right)\left(x_{F}-x\right)}{x_{F}-x_{E}}\right\}^{1 / 2}, & x \geq x_{E},\end{cases}
$$

From equation (5) it is clear that $Z(x)$ depends on three parameters;

$$
p_{1}=\beta\left(T_{E}-T_{0}\right), \quad p_{2}=\mu, \quad p_{3}=\beta\left(T_{F}-T_{0}\right) .
$$

\subsection{Evaluation of force and particle velocity}

The force and particle velocity at the heated end of the main rod were obtained from the measured strains $\varepsilon_{A}(t)$ and $\varepsilon_{B}(t)$ at $x_{A}$ and $x_{B}$, respectively, by use of the method developed by Lundberg et. al. [2]. In this method the rod is assumed to be linearly-elastic with Young's modulus $E$, density $\rho$ and cross- 
section area $A$. The wave propagation speed $c(x)=(E / \rho)^{1 / 2}$ and the characteristic impedance $Z(x)=A(E \rho)^{1 / 2}$ depends on the axial co-ordinate $x$. For convenience these two functions are modelled as piece-wise constant. Thus, the rod $x_{0}<x<x_{n}$ is represented by the wave propagation speed $c_{j}$ and the characteristic impedance $Z_{j}$ in the intervals $x_{j-1}<x<x_{j}$ for $j=1,2, \ldots, n$, where $x_{0}=x_{A}$ and $x_{n}=x_{F}$, i. e. the heated end inside the furnace. The continuous variation in the rod can be described with desired accuracy by the use of a sufficient number of segments in the model. The present model consists of 40 segments. The first segment is between $x_{A}$ and $x_{B}$ and the rest have a constant length $\left(x_{j}-x_{j-1}=\left(x_{F}-x_{B}\right) / 39\right)$. The discrete model is related to the characteristic impedance function $Z(x)$ through $Z_{j}=\left[Z\left(x_{j-1}\right) Z\left(x_{j}\right)\right]^{1 / 2}$. As each segment conserves its mass the wave propagation speed is $c_{j}=Z_{j} / A_{0} \rho_{0}$, where $A_{0}$ and $\rho_{0}$ are the values at room temperature.

The normal force at $x_{A}$ and $x_{B}$ are related to measured strains through

$$
N_{A}(t)=A E \varepsilon_{A}(t), \quad N_{B}(t)=A E \varepsilon_{B}(t),
$$

respectively. The particle velocity $v_{A}(t)$ at $x_{A}$ are related to these quantities by

$$
v_{A}(t)=v_{A}\left(t_{P}\right)+\frac{1}{Z_{1}}\left[-N_{A}(t)-N_{A}\left(t_{p}\right)+2 N_{B}\left(t-t_{B A}\right)\right]
$$

where $t_{P}=t-2 t_{B A}, t_{B A}=\left(x_{B}-x_{A}\right) / c_{1}$. From $N_{A}(t)$ and $v_{A}(t)$ the corresponding quantities $N_{j}(t)$ and $v_{j}(t)$ at the cross-section $x_{j}$ can be determined by repeated use $(j=1,2, \ldots, n)$ of the relations

$$
\begin{aligned}
& N_{j}(t)=\frac{1}{2}\left[N_{j-1}\left(t+t_{j}\right)+N_{j-1}\left(t-t_{j}\right)\right]+\frac{Z_{j}}{2}\left[v_{j-1}\left(t+t_{j}\right)-v_{j-1}\left(t-t_{j}\right)\right], \\
& v_{j}(t)=\frac{1}{2}\left[v_{j-1}\left(t+t_{j}\right)+v_{j-1}\left(t-t_{j}\right)\right]+\frac{1}{2 Z_{j}}\left[N_{j-1}\left(t+t_{j}\right)-N_{j-1}\left(t-t_{j}\right)\right],
\end{aligned}
$$

respectively, where $t_{j}=\left(x_{j}-x_{j-1}\right) / c_{j}$. Finally the force $F(t)$ and particle velocity $v(t)$ at the heated end of the rod can be expressed as

$$
F(t)=-N_{n}(t), \quad v(t)=v_{n}(t),
$$

respectively. The force and particle velocity at the impacted end of the extension rod are related to the measured strain $\varepsilon_{C}(t)$ at $x_{C}$ through

$$
F(t)=-A E \varepsilon_{C}\left(t+t_{C}\right), \quad v(t)=-c \varepsilon_{C}\left(t+t_{C}\right),
$$

respectively, where $t_{C}=L_{C} / c$ and $L_{C}$ is the distance between the impacted end of the extension rod and cross-section $x_{C}$. The transmitted pulse at $x_{C}$ is not superimposed by any reflections since the extension rod is uniform and long enough for the studied time interval.

\subsection{Minimization procedure}

By the introduction of the parameter vector $p=\left\{p_{1}, p_{2}, p_{3}\right\}$ the characteristic impedance can be written as $Z(x)=\widetilde{Z}(x, p)$ and the evaluated force at the free end of the rod becomes $F(t)=\widetilde{F}(t, p)$. As the real force is identically zero the error, $\xi(p)$, is defined as the time integral of the squared evaluated force, i. e.

$$
\xi(p)=\int_{0}^{t_{T}} \widetilde{F}^{2}(t, p) d t
$$

where $t_{T}$ is the studied time interval. The problem to evaluate the characteristic impedance function is then reduced to find the vector $p$ that minimizes the integral. To solve this problem a method developed by Popper and Csizmas [5], which is a version of the generalized secant method, has been used. This minimization procedure is presented in detail in the appendix. 


\subsection{Experimental set-up}

Experimental tests were performed with the set-up shown in Figure 2. The end part of the main rod was heated by a furnace to $950^{\circ} \mathrm{C}$. The main rod was impacted by a cylindrical steel hammer driven by an air gun. To avoid the influence of 3-D wave propagation effects a damper between the hammer and the main rod was used. This gives a smoother incident pulse. The main rod is made of a refractory austenitic steel (Thyssen Thermax 4841) with wave propagation speed $4935 \mathrm{~m} / \mathrm{s}$, density $7830 \mathrm{~kg} / \mathrm{m}^{3}$ and elastic limit $230 \mathrm{MPa}$. The extension rod is made of high strength steel (Marval $18 \mathrm{H}$ ) with wave propagation speed $4815 \mathrm{~m} / \mathrm{s}$, density $8040 \mathrm{~kg} / \mathrm{m}^{3}$ and elastic limit $1800 \mathrm{MPa}$. The hammer is made of the same material as the main rod and its diameter is the the same as that of the test rods, i. e. $20 \mathrm{~mm}$. The length of the hammer is $200 \mathrm{~mm}$. The test rods were supported by low-friction slide bearings so they were axially movable during the tests.

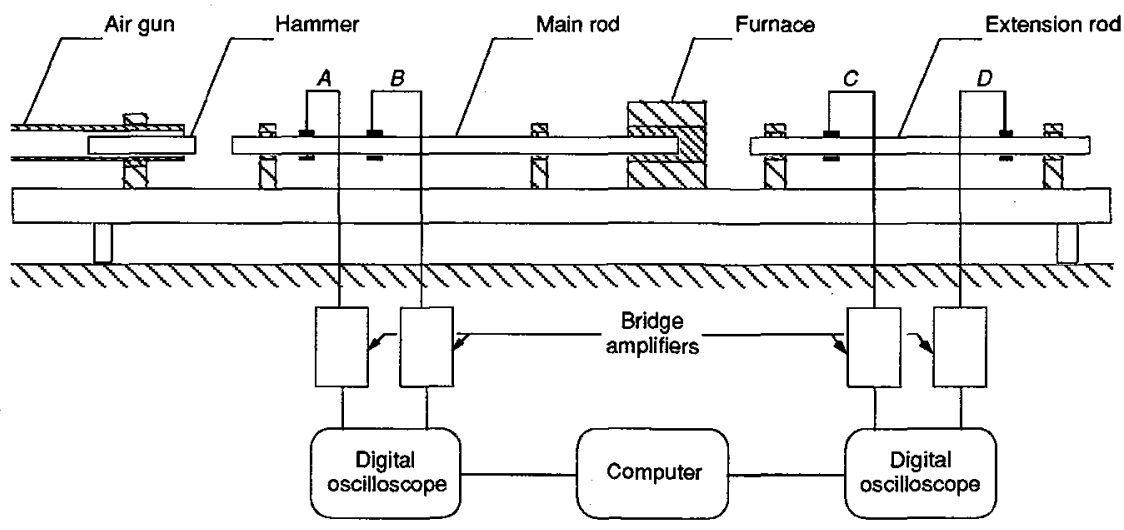

Figure 2. Experimental set-up.

The axial strains were recorded at the cross-sections $A$ and $B$ of the main rod during the tests with a free end. During the impact test the strain at the cross-section $C$ of the extension rod was also recorded. Two pairs of strain gauges (Micro-Measurements CEA-06-125UT-120) were bonded to each of the cross-sections $A, B, C$ and $D$ as shown in Figure 3 . The gauges were connected to bridge amplifiers (Tektronix AM 502) in such a way that contributions to the strains from small accidental bending were suppressed. The strain signals were recorded by means of two two-channel digital oscilloscopes (LeCroy 9400) with a sampling interval of $1 \mu \mathrm{s}$. The recorded strain histories were transferred to a computer (Macintosh II) for evaluation. The channels for strain measurement were rigourously balanced by making use of the fact that the momentum in the incident pulse is constant in the rod system. The impact velocity, $V$, was calculated from the measured momentum of the incident pulse and was found to be approximately $3 \mathrm{~m} / \mathrm{s}$ during the test with a free end and $5 \mathrm{~m} / \mathrm{s}$ during the impact test. The wave propagation speed at room temperature, $c_{0}$, was found from preliminary tests. During these tests use was made of the strain recorded at cross-section $D$ of the extension rod.

(a)

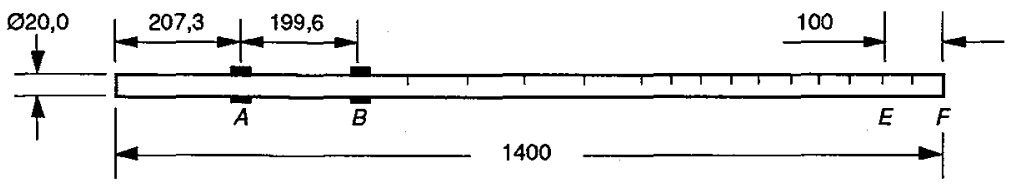

(b)

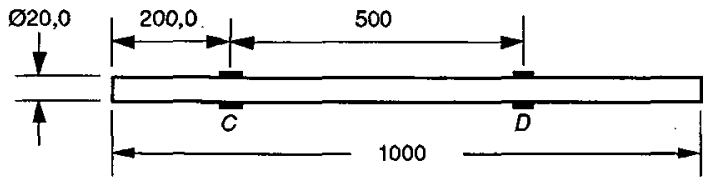

Figure 3. Test rods. (a) Main rod. (b) Extension rod. 


\section{Results}

The method was used to find the characteristic impedance function for the rod with the rod end heated to $950^{\circ} \mathrm{C}$. The initial approximation of the characteristic impedance function was $Z(x)=Z_{0}$ and the evaluated characteristic impedance function shown in figure 4 was found after 34 iterations. The method has also been tested with other initial conditions and it converges to the same solution.

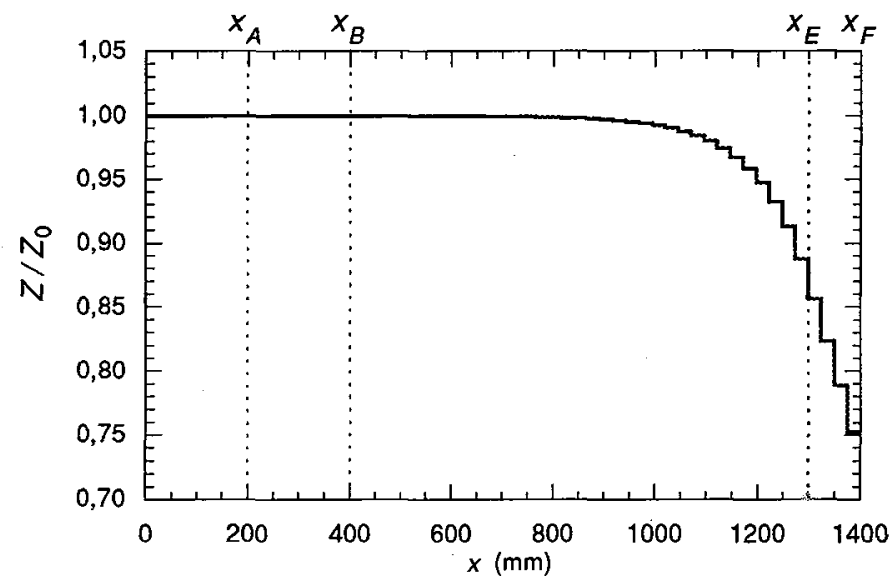

Figure 4. Evaluated characteristic impedance $Z / Z_{0}$ vs. axial co-ordinate $x$. Rod end at $950^{\circ} \mathrm{C}$.

The accuracy of the method can be defined as the accuracy in the evaluated force and particle velocity at the end of the rod. With a free end the evaluated force, $F_{\text {free }}(t)$, should be identically zero and a practical requirement is that $F_{f r e e}(t)$ is much smaller than $F_{f i x}(t)$ which would be obtained with fixed end. $F_{f i x}(t)$ is of the magnitude $Z_{0} V$ during the reflection of the incident pulse and this gives the requirement $\left|F_{\text {free }}(t) / Z_{0} V\right| \ll 1$. In figure 5 the dimensionless force $F_{\text {free }}(t) / Z_{0} V$ obtained with the initial, (a), and evaluated, (b), characteristic impedance for the heated rod and the dimensionless force obtained for a rod at room temperature, (c), are compared. During these tests no damper was used.
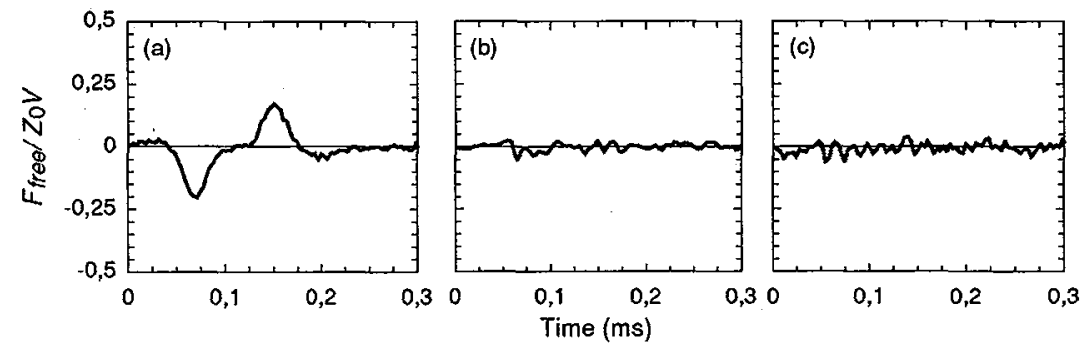

Figure 5. Dimensionless force $F_{\text {free }} / \mathrm{Z}_{0} \mathrm{~V}$ at free end $v$ s time:(a) Rod end at $950^{\circ} \mathrm{C}$, initial characteristic impedance, (b) Rod end at $950^{\circ} \mathrm{C}$, evaluated characteristic impedance, (c) Rod end at room temperature $24^{\circ} \mathrm{C}$.

As a second validation of the method a split Hopkinson impact test was made. In this test the contact force between the main rod, heated to $950{ }^{\circ} \mathrm{C}$, and the extension rod, held at $24{ }^{\circ} \mathrm{C}$ was evaluated. The velocities of the two end surfaces were also evaluated. The results obtained from measurement of strains at $A$ and $B$ on the main rod were compared with those obtained from measurement of strain at $C$ on the extension rod. To prevent heating of the extension rod it was put in contact with the main rod just before impact. In order to have more realistic conditions the impact velocity was increased to $5 \mathrm{~m} / \mathrm{s}$ and the impact was made without damper. This increases both the strain and the strain-rate in the incident pulse and the evaluation of force and velocity is more difficult. The results from this test is shown in figure 6. 
(a)
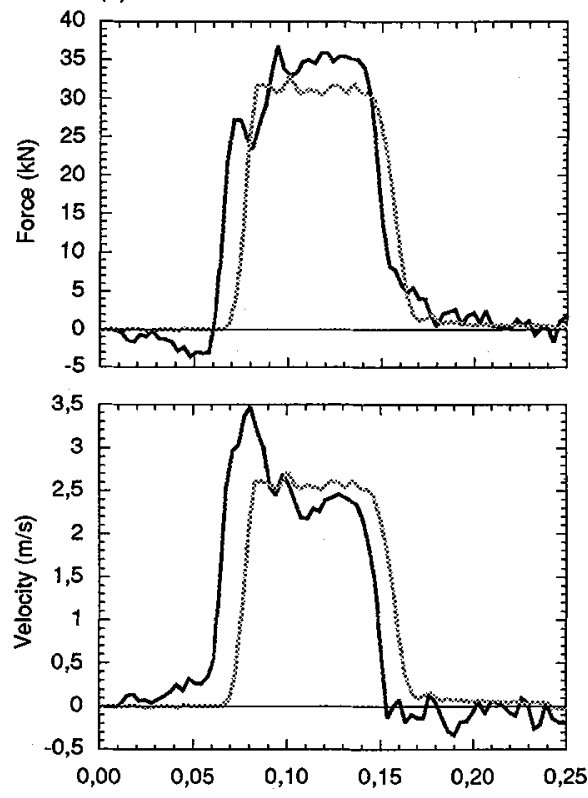

(b)
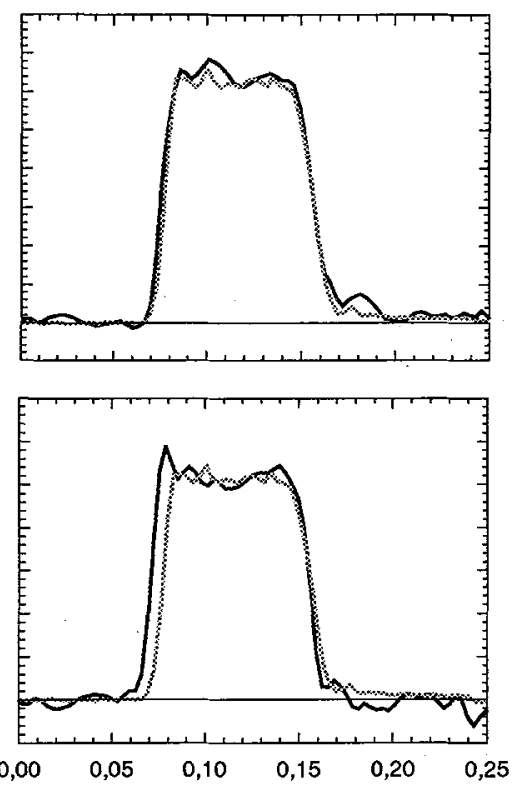

Time (ms)

Figure 6. Contact force and particle velocity at rod interface vs, time obtained from measurement of strains at $A$ and $B$ (solid line) and at $C$ (dotted line). Main rod end at $950^{\circ} \mathrm{C}$, extension rod end at $24^{\circ} \mathrm{C}$. (a): initial characteristic impedance. (b): evaluated characteristic impedance.

\section{Discussion}

The method has shown to be stable with a reasonable initial assumption and the errors are reduced to the same low level as for tests with rods with constant characteristic impedance as shown in figure 5 for free end tests and in figure 6 for an impact test. The reduction of the errors for times before impact is considerable. Also to be noted is the small discrepancy between the two velocities in figure 6.b during the rise time of the pulse, this is due to an imperfect contact between the two surfaces and corresponds to a relative displacement of $10 \mu \mathrm{m}$. The temperature distribution, $T(x)$, remains unknown. This is normally not a problem. The temperature distribution can be calculate if the relation between the Young's modulus and the temperature is known. It will, however, be less accurate than the characteristic impedance function. The method can be extended to a more complex heating of the rod where other assumptions on the characteristic impedance function can be used. In general the characteristic impedance can be expressed as a polynomial of order $n$ and the method can be generalized to find a set of $n$ parameters. With this method several different techniques using 1-D wave propagation can be used at high temperatures, e. g. the split Hopkinson bar technique, threepoint bend tests and mobility measurements for structures.

\section{Acknowledgements}

The authors wish to thank Professor Bengt Lundberg for his contribution to this work. They are also indebted to the Universities of Lulea and Bordeaux I, the Ministère de la Recherche et de la Technologie and the Région Aquitaine for their financial support.

\section{References}

[1] J. D. Campbell, Mechanisms of dynamic behaviour of materials. Progress Report $n^{\circ} 3$, Dept. Engg. Sci. Univ. of Oxford, (1971).

[2] B. Lundberg, J. Carlsson and K. G. Sundin, Analysis of elastic waves in non-uniform rods from two-point strain measurement. Journal of Sound and Vibration 137, 483-493 (1990).

[3] M. M. Sondhi and B. Gopinath, Determination of vocal-tract shape from impulse response at the lips. J. Acoust. Soc. Am. 49, 1867-1873 (1971). 
[4] B. Lundberg and M. Lesser, Determination of the distributed parameters of a lossless wave supporting system from boundary measurements. Appl. Sci. Res. 33, 151-162 (1977).

[5] G. Popper and F. Csizmas, Minimization by the secant method, an application to identification of frames. Acta Technica Acad. Sci. Hung. 100, 259-267 (1987).

[6] G. H. Golub and C. Reinsch, Singular value decomposition and least square solutions. Numer. Math. 14, 403420 (1970).

\section{Appendix}

The problem is to find the vector $p$ that minimizes the time integral of the squared evaluated force (12). A vector representation of the force is $F(p)$ with the elements $F_{n}(p)=\widetilde{F}(n \Delta t, p)$. The error can then be written as

$$
\xi(p)=\Delta t \sum_{n=1}^{m} F_{n}^{2}(p)=\Delta t F^{T}(p) F p ，
$$

where $\Delta t$ is the sample rate and $m$ the number of samples. Let $p^{1}, p^{2}, p^{3}$ and $p^{4}$ be given approximations of the minimizer, $p$, of the functional (A.1). A 3-dimensional plane which allocates $F(p)$ at 4 points has the equation

$$
\left\{\begin{array}{c}
p \\
y(p)
\end{array}\right\}=\left\{\begin{array}{c}
p^{1} \\
F\left(p^{1}\right)
\end{array}\right\}+\left\{\begin{array}{c}
p^{2}-p^{1} \\
F\left(p^{2}\right)-F\left(p^{1}\right)
\end{array}\right\} q_{1}+\left\{\begin{array}{c}
p^{3}-p^{1} \\
F\left(p^{3}\right)-F\left(p^{1}\right)
\end{array}\right\} q_{2}+\left\{\begin{array}{c}
p^{4}-p^{1} \\
F\left(p^{4}\right)-F\left(p^{1}\right)
\end{array}\right\} q_{3}
$$

where $p^{\mathrm{k}} \in R^{3}, F\left(p^{\mathrm{k}}\right) \in R^{\mathrm{m}}$ and $y(p)$ is an approximation of $F(p)$. The functional (A.1) can be replaced by the functional $y^{T}(q) y(q)$

and its minimizer, $q=\left\{q_{1}, q_{2}, q_{3}\right\}$ can be used to calculate the next approximation $p^{5}$ of the minimizer $p$. The second equation in (A.2) can be written as

$$
y=b-A q
$$

with $b=F\left(p^{1}\right)$ and $A$ as the mx3 matrix; $A=\left[F\left(p^{1}\right)-F\left(p^{2}\right), F\left(p^{1}\right)-F\left(p^{3}\right), F\left(p^{1}\right)-F\left(p^{4}\right)\right]$. The minimizer $q$ to equation (A.3) is the least square solution of the linear system of equations

$$
A q=b
$$

If the rank of $A$ is less than three the solution is not unique and the vector with minimum length has been chosen . This vector is

$$
q=A^{+} b
$$

where $A^{+}$is the pseudo-inverse of $A$. By the singular value decomposition the matrix $A$ can be factorized as

$$
A=U \Sigma V^{T}
$$

For the decomposition of $A$ a Pascal version of the procedure SVD by Golub and Reinsch [6] has been used . The diagonal matrix $\Sigma=\left\langle\sigma_{1}, \sigma_{2}, \sigma_{3}\right\rangle$, with $\sigma_{1} \geq \sigma_{2} \geq \sigma_{3}$, contains the singular values of $A$ and to get a numerically stable pseudo-inverse it is necessary to equate the small singular values to zero. The pseudo-inverse of $\Sigma$ has been defined as $\Sigma^{+}=\left\langle\sigma_{1}{ }^{+}, \sigma_{2}{ }^{+}, \sigma_{3}{ }^{+}\right\rangle$ with the diagonal elements

$$
\sigma_{i}^{+}=\left\{\begin{array}{cc}
1 / \sigma_{i} & \text { for } \sigma_{i}>\sigma_{1} \cdot 10^{-4} \\
0 & \text { for } \sigma_{i}<\sigma_{1} \cdot 10^{-4}
\end{array}\right.
$$

This gives the minimizer, $q$, to equation (A.3)

$$
q=V \Sigma^{+} U^{T} b
$$

The next approximation to $p$ follows then from the first equation in (A.2)

$$
p^{5}=\left(1-q_{1}-q_{2}-q_{3}\right) p^{1}+q_{1} p^{2}+q_{2} p^{3}+q_{3} p^{4}
$$

Thus, the first iteration has been completed. To continue the iterations the approximation which gives the greatest error was replaced with $\boldsymbol{p}^{5}$. As a stop criterion

$$
\frac{s(\xi)}{m(\xi)}<10^{-3}
$$

was used, where $m(\xi)$ is the average error

$$
m(\xi)=\frac{1}{4} \sum_{i=1}^{4} \xi\left(p^{i}\right)
$$

and $s(\xi)$ is the standard deviation

$$
s(\xi)=\left[\frac{1}{3} \sum_{i=1}^{4}\left(\xi\left(p^{i}\right)-m(\xi)\right)^{2}\right]^{1 / 2}
$$

Based on physical considerations the parameters are limited to certain ranges of values. From equation (3) follows that $0<$ $\beta\left(T_{2}-T_{0}\right)<1$ if the Young's modulus is to be a decreasing function of temperature and positive throughout the rod. Further $T_{2}>T_{1}$ and $\mu>0$ since the furnace is the only heat source in the system. This gives the following ranges for the parameters

$$
0<p_{1}<p_{3}<1, \quad 0<p_{2}<\infty
$$

These limits have been checked for each iteration step and the parameters have been adjusted to the closest limit if necessary. As the four initial approximations

$$
p^{1}=\left\{\begin{array}{l}
0 \\
0 \\
0
\end{array}\right\}, p^{2}=\left\{\begin{array}{c}
0.1 \\
0 \\
0
\end{array}\right\}, p^{3}=\left\{\begin{array}{c}
0 \\
0.1 \\
0
\end{array}\right\}, p^{4}=\left\{\begin{array}{c}
0 \\
0 \\
0.1
\end{array}\right\}
$$

were used, where $p^{1}$ corresponds to a rod with constant characteristic impedance. 